\title{
Inflammatory pseudotumoural endotracheal mucormycosis with cartilage damage
}

\author{
L-C. Luo, D-Y. Cheng, H. Zhu, X. Shu and W-B. Chen
}

ABSTRACT: Mucormycosis is a rare opportunistic infection usually associated with immunosuppression, diabetes mellitus or haematological malignancy. Herein, we report an unusual case of mucormycosis in a 46-yr-old male patient with diabetes presenting with an endotracheal mass obstructing the trachea and cartilage damage. Histological examination of the bronchoscopy biopsy specimens revealed invasive mucormycosis. The patient was treated with intravenous amphotericin B followed by removal of the lesion via bronchoscopy.

\section{KEYWORDS: Cartilage damage, dyspnoea, mucormycosis, tracheal stenosis}

$\mathbf{M}$ ucormycosis, also called zygomycosis, is an important opportunistic infection caused by fungus that belongs to the class Zygomycetes, which is the third most common invasive fungal infection after candidosis and aspergillosis [1]. Although the infection rate of mucormycosis is very low, patients with immunosuppression, diabetes mellitus or haematological malignancy are at the highest risk for mucormycosis. Mucorales rarely invades the trachea. Tracheal cartilage damage is extremely rare in pulmonary mucormycosis. Herein, we report an unusual case of mucormycosis in a diabetic male presenting with an endotracheal mass obstructing the trachea and cartilage damage with histological confirmation of endotracheal mucormycosis.

\section{CASE REPORT}

A 46-yr-old male nonsmoker was admitted to our hospital (West China Hospital, Sichuan University, Chengdu, China) because of throat discomfort, cough and expectoration of 1 month duration. Simultaneously, he had mild dyspnoea, especially during exercise. He also had type 2 diabetes, which had been diagnosed $\sim 5$ yrs previously; his diabetes had been poorly controlled by diet alone. Physical examination revealed that bilateral lung breathing sounded rough and moist rales could be heard in the left lung. Laboratory data showed that the white blood cell count was $11.82 \times 10^{9} \mathrm{~L}^{-1}$ with $76.7 \%$ neutrophils and hyperglycemia $\left(18.57 \mathrm{mmol} \cdot \mathrm{L}^{-1}\right)$ was observed. Blood urea nitrogen and creatinine were normal. Acid-fast bacilli smears and cultures of sputum were negative. We also excluded the possibility of HIV infection. Computed tomography of the chest and neck indicated that the tracheal wall was thickened, the cricoid cartilage was damaged and the lumen was narrow (fig. 1). Fibreoptic bronchoscopy revealed that the upper airway mucosal was oedematus, thickened and that the lumen was narrow and funnel-shaped. A valve-like neoplasma was seen on the right wall of upper trachea (fig. 2). Biopsies were obtained by removal of the lesion via bronchoscopy and the histological sections revealed moderate chronic inflammation in the mucosa of the upper trachea, associated with inflammatory exudates, necrosis, granulation tissue proliferation and numerous hyphae of mucormycosis. Histochemical staining indicated negative acid-fast stain, positive periodic acidSchiff, positive hexamine stain and positive mucus carmine stain. He was diagnosed with endotracheal mucormycosis with granulation formation and diabetes mellitus.

Diabetic control was achieved with insulin, and amphotericin B (AMB) was administered intravenously for mucormycosis. The dose of AMB was gradually increased from $5 \mathrm{mg} \cdot \mathrm{day}^{-1}$ to $40 \mathrm{mg} \cdot$ day $^{-1}$. However, during the course of treatment our patient experienced adverse reactions of shivering, high fever (the highest temperature was $39.5^{\circ} \mathrm{C}$ ) and pain in his right forearm. As a result, we had to decrease the dose
AFFILIATIONS

Dept of Respiratory Medicine, West China Hospital, Sichuan University, Chengdu, China.

CORRESPONDENCE

D-Y Cheng

Dept of Respiratory Medicine,

West China Hospital

Sichuan University

Chengdu 610041

China

E-mail: chengdeyun@sohu.com

Received:

Feb 262009

Accepted after revision:

May 022009

PROVENANCE

Submitted article, peer reviewed. 


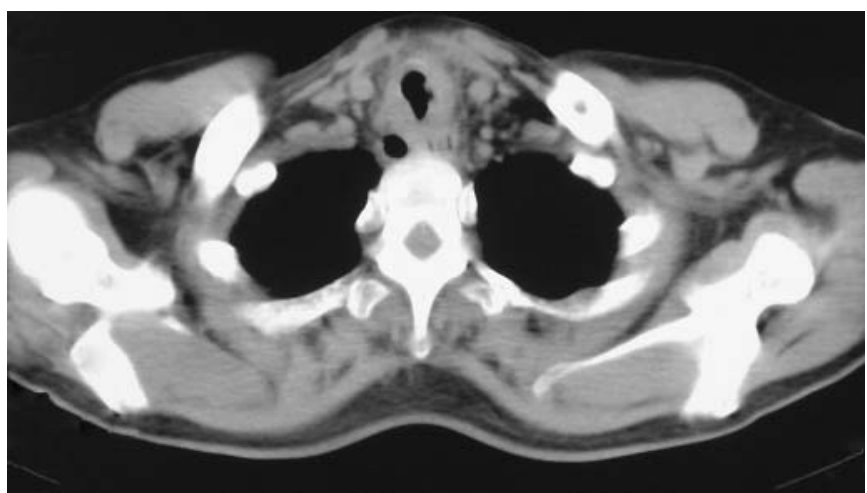

FIGURE 1. A computed tomography scan indicating that the tracheal wall was thickened, the cricoid cartilage was damaged and the lumen was narrow.

of $\mathrm{AMB}$ to $35 \mathrm{mg} \cdot \mathrm{day}^{-1}$ and added $5 \mathrm{mg}$ of dexamethasone to the i.v. drip, simultaneously with aerosolised AMB inhalation three times a day (the daily dose was $25 \mathrm{mg}$ ). Fibreoptic bronchoscopy was repeated 15 days after admission and showed necrotic tissue on the right anterior wall of the trachea (fig. 3). After clamping out the necrotic tissue with biopsy forceps, we found the structure of his tracheal cartilage was damaged (fig. 4). The dose of AMB was gradually increased to $50 \mathrm{mg} \cdot$ day $^{-1}$. When the total dose of AMB administered reached $980 \mathrm{mg}$, the patient's blood urea nitrogen increased to $22.47 \mathrm{mmol} \cdot \mathrm{L}^{-1}$ and creatinine increased to $215.6 \mu \mathrm{mol} \cdot \mathrm{L}^{-1}$. Thus, i.v. liposomal AMB of $50 \mathrm{mg} \cdot$ day $^{-1}$ was substituted for $\mathrm{AMB}$, and he also received Chinese traditional patent drugs for oral use to improve his kidney function. The blood urea nitrogen level was $8.12 \mathrm{mmol} \cdot \mathrm{L}^{-1}$ and creatinine was $107.7 \mu \mathrm{mol} \cdot \mathrm{L}^{-1} 5$ days after i.v. liposomal AMB. Following this, we switched to AMB for treatment. After nearly 2 months of residential treatment with a total of $1.8875 \mathrm{~g}$ i.v. AMB and $0.4 \mathrm{~g}$ liposomal AMB, fibreoptic bronchoscopy showed that the patient's tracheal neoformative mucosa had covered the damaged tracheal cartilage; thus, tracheal mucosa was smooth and the airway was unobstructed (fig. 5). After being discharged, the patient was still asked to continue treatment with inhaled atomised AMB.

\section{DISCUSSION}

Mucorales, which belong to the subclass Zygomycetes of the class Phycomycetes, are opportunistic fungi that cause deep tissue infection. The production of spores, which become airborne, leads to the primary route of inoculation in the respiratory tract. The spores of mucorales show minimal pathogenicity in immunocompetent hosts, because the macrophages can kill spores by phagocytosis and oxidative killing mechanisms. However, macrophages in a host with immune deficiency or diabetes can lose their ability to inhibit spore germination and prevent the hyphae and spores from invading the bronchus and lung [2]. Mucormycosis is usually seen in diabetic patients with or without ketoacidosis, or in patients with other risk factors, including cancer (such as leukaemia, lymphoma, multiple myeloma, etc.), AIDS, chemotherapy, organ transplantation, anti-rejection treatment, iron chelate treatment, severe malnutrition and the application of broad-spectrum antibiotics [3]. Clinically observed forms of mucormycosis are rhinocerebral, pulmonary, cutaneous, gastrointestinal, disseminated and other

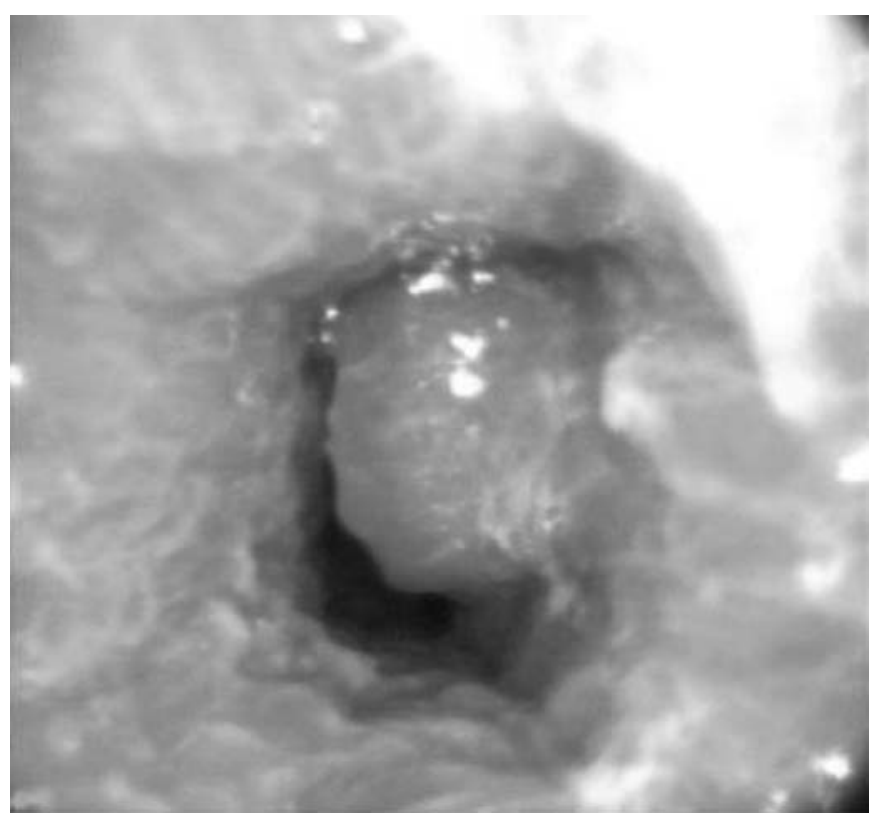

FIGURE 2. Fibreoptic bronchoscopy revealed a valve-like neoplasma on the right wall of the upper trachea, $\sim 1.5 \mathrm{~cm}$ from the glottis.

clinical manifestations. The most commonly involved mucormycosis are rhinocerebral and pulmonary forms [4].

The clinical manifestations of pulmonary mucormycosis infection cannot be easily distinguished from those of pulmonary bacterial infection. Patients may present with fever, cough, expectoration, haemoptysis and pleuritic chest pain. The most prominent physical finding is bilateral pulmonary fixed rales or pleural rubs. The laboratory examination results are nonspecific, with most patients only showing an increase in their white blood cell count. The radiographic presentations of patients with pulmonary mucormycosis appear abnormal and include infiltrate, cavity, consolidation, air crescent sign, pleural effusions, fistula, pneumothorax and pulmonary collapse. There are particular clinical features, such as airway obstruction, when mucorales invade the trachea. Lee et al. [5] reviewed the literature and reported that only 87 patients had been reported with localised pulmonary mucormycosis. Of these, 34 patients had evidence of visible endobronchial disease. DONOHUE [6] has reported that there is an additional, distinct clinical syndrome of mucormycosis in diabetic patients, which is an endobronchial lesion. In these patients, the disease manifests as airway obstruction but chest radiography shows mediastinal widening or pulmonary atelectasis. Bronchoscopy appears to be a successful diagnostic modality in diagnosing mucormycosis with major airway involvement. Bronchoscopy reveals granulation tissue and grey-white mucoid material that frequently blocks a major airway. The airways involved are typically oedematous and necrotic, or lesions with an appearance suggestive of a bronchial adenoma. HUSARI et al. [7] postulated that a submucosal, invasive fungal infection caused a submucosal abscess, which presented as an endobronchial mass. Simultaneously, it may lead to tracheal cartilage damage as in the unusual case presented herein, which is extremely rare in pulmonary mucormycosis and has not been reported previously. 


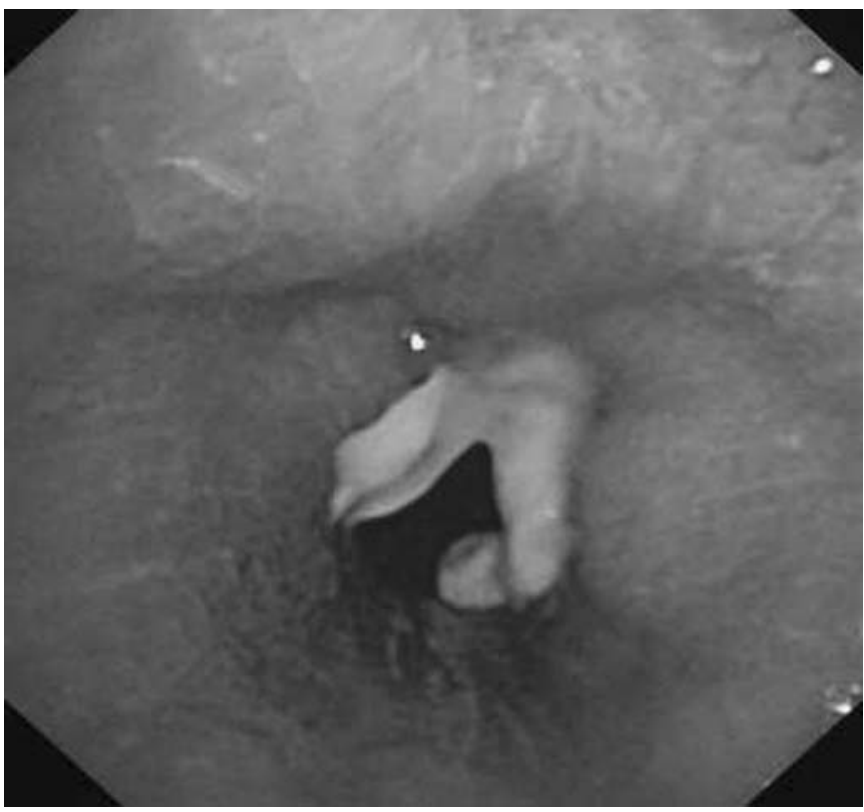

FIGURE 3. Fibreoptic bronchoscopy revealed necrotic tissue on the right anterior wall of trachea, $\sim 1.5 \mathrm{~cm}$ from the glottis.

In addition to presenting with cough and expectoration, our patient had shortness of breath prior to admission, suggesting that there was airway obstruction that led to dyspnoea. When we performed the first fibreoptic bronchoscopy, without the pathological evidence, we preliminarily considered the lesion as endotracheal tuberculosis. After clamping of the necrotic tissue with biopsy forceps, we found that the structure of his tracheal cartilage was damaged. We didn't remove the protruding cartilage as we thought it might cause further damage, such as tracheal fistula. In addition, patients with tracheal cartilage damage can sometimes expectorate cartilage spontaneously [8].

A definite diagnosis of pulmonary mucormycosis can only be made with fungal aetiology and histopathology results. Sputum culture, used as a simple preliminary diagnostic method, is often negative. The correct diagnosis is usually made by histopathology. Histopathological specimens of lesions can be collected by bronchoscopy biopsy, percutaneous needle biopsy or open lung biopsy; all of which show tissue invasion with characteristic broad, nonseptate hyphae with right-angle branching [9]. As demonstrated by the bronchoscopy biopsy, our patient was diagnosed with confirmed mucormycosis.

As reported in the literature, $\mathrm{AMB}$ remains the primary antimicrobial agent with evidence of antifungal efficacy in mucormycosis [10]. AMB can bind to ergosterol incorporated in fungal cellular membranes, resulting in disorganisation of the membrane and formation of specific pores or channels. These defects cause depolarisation of the membrane and an increase in membrane permeability to intracellular important substances such as potassium and nucleotides, amino acids leakage then fungal cell death [11]. Clinical use of AMB is limited by frequent adverse reactions, including chills, fever, nausea, vomiting, hepatotoxicity and nephrotoxicity. Therefore, AMB should be

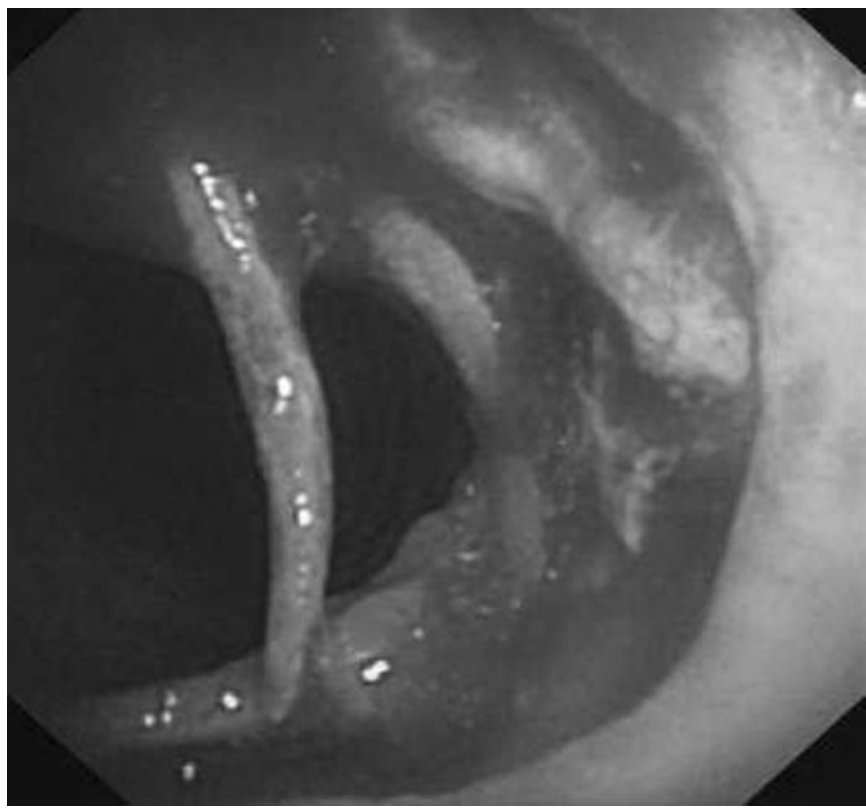

FIGURE 4. After clamping out of necrotic tissue with biopsy forceps, the structure of the patient's tracheal cartilage was found to be damaged.

administered in small doses, which increase gradually and via a slow i.v. drip. The usual dose of AMB is up to $1-1.5 \mathrm{mg} \cdot \mathrm{kg}^{-1} \cdot \mathrm{day}^{-1}$ and therapy should be continued for at least 6-8 weeks [2] During treatment the patients' hepatic and renal function should be monitored, and any adverse reactions should be actively dealt with. Patients with renal insufficiency may inhale atomised AMB as a joint treatment [12]. Liposomal AMB could potentially be used when patients experience intolerable symptoms during infusion from conventional AMB. Pharmacokinetic studies have shown that a smaller volume of distribution and higher serum

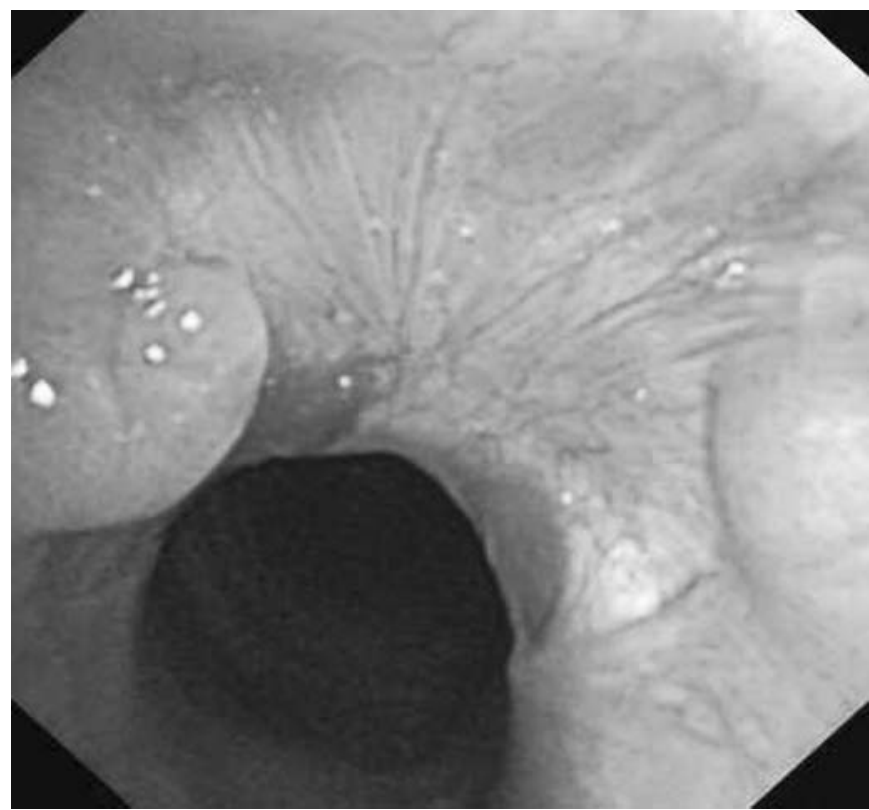

FIGURE 5. Following amphotericin B therapy, the tracheal mucosa was smooth and the airway was unobstructed. 
levels than conventional AMB improve efficacy and reduce adverse reactions [13]. Other antifungal agents, such as triazole drugs, have no antifungal activity on mucorales, except posaconazole, which is effective at reducing fungal burden in organs [14]. Because of the aggressive nature of the disease when vascular invasion and infarction of tissue are present, and the difficulty in eradicating these fungi by medical treatment only, surgical therapy is crucial and highly recommended and must be performed without delay. Simultaneously, control of the patient's underlying disease, such as diabetes mellitus, is also important to increase survival rate.

Endotracheal involvement is a rare presentation of mucormycosis, which lacks specific clinical manifestations. When patients with diabetes mellitus or haematological malignancy have dyspnoea or tracheal stenosis, these cases should be highly suggestive of possible mucormycosis, and patients should receive fibreoptic bronchoscopy. A combination of antimicrobial treatment and surgery offers patients the best chance of a cure.

\section{STATEMENT OF INTEREST}

None declared.

\section{REFERENCES}

1 Eucker J, Sezer O, Graf B, et al. Mucormycosis. Mycoses 2001; 44: 253-260.
2 Chayakulkeeree M, Ghannoum MA, Perfect JR. Zygomycosis: the re-emerging fungal infection. Eur J Clin Microbiol Infect Dis 2006; 25: 215-229.

3 Aboutanos MB, Joshi M, Scalea TM. Isolated pulmonary mucormycosis in a patient with multiple injuries: a case presentation and review of the literature. J Trauma 2003; 54: 1016-1019.

4 Bigby TD, Serota ML, Tierney LM, et al. Clinical spectrum of pulmonary mucormycosis. Chest 1986; 89: 435-439.

5 Lee FY, Mossad SB, Adal KA. Pulmonary mucormycosis: the last 30 years. Arch Intern Med 1999; 159: 1301-1309.

6 Donohue JF. Endobronchial mucormycosis. Chest 1983; 83: 585.

7 Husari AW, Jensen WA, Kirsch CM, et al. Pulmonary mucormycosis presenting as an endobronchial lesion. Chest 1994; 106: 1889-1891.

8 Park MJ, Woo IS, Son JW, et al. Endobronchial tuberculosis with expectoration of tracheal cartilages. Eur Respir J 2000; 15: 800-802.

9 Li N, Carter D. Pulmonary mucormycosis: report of two cases and review of the literature. Pathol Case Rev 2003; 8: 275-278.

10 Thomas R, Christopher DJ, Roy A, et al. Pulmonary mucormycosis presenting as endobronchial tumours - case reports. Respir Med Extra 2005; 1: 27-30.

11 Brajtburg J, Powderly WG, Kobayashi GS, et al. Amphotericin B: current understanding of mechanisms of action. Antimicrob Agents Chemother 1990; 34: 183-188.

12 Furco A, Mouchet B, Carbonnelle M, et al. Pulmonary mucormycosis: benefit of aerosol amphotericin B. Rev Mal Respir 2001; 18: 309-313.

13 Heinmann V, Bosse D, Jehn U, et al. Pharmacokinetics of liposomal amphotericin B (AmBisone) in critical ill patients. Antimicrob Agents Chemother 1997; 41: 1275-1280.

14 Rogers TR. Treatment of zygomycosis: current and new options. J Antimicrob Chemother 2008; 61: Suppl. 1, i35-i39. 